\title{
A COMBINATORIAL IDENTITY FOR THE SUM OF DIVISORS FUNCTION INVOLVING $p_{\mathbf{r}}(\mathbf{n})$
}

\author{
Sumit Kumar Jha \\ International Institute of Information Technology, Hyderabad, India \\ kumarjha.sumit@research.iiit.ac.in \\ Received:, Revised: , Accepted: , Published:
}

\begin{abstract}
Let $\sum_{d \mid n} d$ denote the sum of divisors of a positive integer $n$, and let $\prod_{j=1}^{\infty}(1-$ $\left.q^{j}\right)^{r}=\sum_{n=0}^{\infty} p_{r}(n) q^{n}$. The aim is of this note is to prove the following interesting combinatorial identity:

$$
\sum_{d \mid n} d=n \sum_{r=1}^{n} \frac{(-1)^{r}}{r}\left(\begin{array}{l}
n \\
r
\end{array}\right) p_{r}(n) .
$$
\end{abstract}

\section{Main result}

In the following, let $\sum_{d \mid n} d$ denote the sum of divisors of a positive integer $n$.

Definition 1 ([3]). The Euler function is the infinite product

$$
E(q):=\prod_{j=1}^{\infty}\left(1-q^{j}\right)=\sum_{n=-\infty}^{\infty}(-1)^{n} q^{\frac{3 n^{2}+n}{2}}
$$

where $|q|<1$.

Definition 2. For any positive integer $r$ define $p_{r}(n)$ by

$$
E(q)^{r}=\prod_{j=1}^{\infty}\left(1-q^{j}\right)^{r}=\sum_{n=0}^{\infty} p_{r}(n) q^{n} .
$$

The function $p_{r}(n)$ is related to the partition function $p(n)$ (which counts the number of partitions of $n$ ), and its congruence properties have been studied like those of the partition function [1].

Our aim is to derive the following combinatorial identity. 
Theorem 1. For all positive integers $n$ we have

$$
\sum_{d \mid n} d=n \sum_{r=1}^{n} \frac{(-1)^{r}}{r}\left(\begin{array}{l}
n \\
r
\end{array}\right) p_{r}(n) .
$$

We require following two lemmas for our proof.

Lemma 1. For all positive integers $n$ we have

$$
\sum_{d \mid n} \frac{1}{d}=\frac{1}{n !} \sum_{k=1}^{n}(-1)^{k}(k-1) ! B_{n, k}\left(E^{\prime}(0), E^{\prime \prime}(0), \ldots, E^{(n-k+1)}(0)\right)
$$

where $E(q)$ is the Euler function, and $B_{n, k} \equiv \mathrm{B}_{n, k}\left(x_{1}, x_{2}, \ldots, x_{n-k+1}\right)$ are the partial Bell polynomials defined by [2, p. 206]

$$
\mathrm{B}_{n, k}\left(x_{1}, x_{2}, \ldots, x_{n-k+1}\right)=\sum_{\substack{1 \leq i \leq n, \ell_{i} \in \mathbb{N} \\ \sum_{i=1}^{n} i i_{i}=n \\ \sum_{i=1}^{n} \ell_{i}=k}} \frac{n !}{\prod_{i=1}^{n-k+1} \ell_{i} !} \prod_{i=1}^{n-k+1}\left(\frac{x_{i}}{i !}\right)^{\ell_{i}} .
$$

Proof. It is easy to see that

$$
\begin{aligned}
\log (E(q)) & =\sum_{j=1}^{\infty} \log \left(1-q^{j}\right) \\
& =-\sum_{j=1}^{\infty} \sum_{l=1}^{\infty} \frac{q^{l j}}{l} \\
& =-\sum_{n=1}^{\infty} q^{n}\left(\sum_{d \mid n} \frac{1}{d}\right) .
\end{aligned}
$$

Let $f(q)=\log q$. Using Faà di Bruno's formula [2, p. 134] we have

$$
\frac{d^{n}}{d q^{n}} f(E(q))=\sum_{k=1}^{n} f^{(k)}(E(q)) \cdot B_{n, k}\left(E^{\prime}(q), E^{\prime \prime}(q), \ldots, E^{(n-k+1)}(q)\right) .
$$

Since $f^{(k)}(q)=\frac{(-1)^{k-1}(k-1) !}{q^{k}}$ and $E(0)=1$, letting $q \rightarrow 0$ in the above equation gives us Equation (2).

Lemma 2. We have, for positive integers $n, k$,

$$
B_{n, k}\left(E^{\prime}(0), E^{\prime \prime}(0), \ldots, E^{(n-k+1)}(0)\right)=\frac{n !}{k !} \sum_{r=1}^{k}(-1)^{k-r}\left(\begin{array}{l}
k \\
r
\end{array}\right) p_{r}(n) .
$$


Proof. We start with the generating function for the partial Bell polynomials as follows:

$$
\begin{aligned}
\sum_{n=k}^{\infty} B_{n, k}\left(E^{\prime}(0), E^{\prime \prime}(0), \ldots, E^{(n-k+1)}(0)\right) \frac{q^{n}}{n !} & =\frac{1}{k !}\left(\sum_{j=1}^{\infty} E^{(j)}(0) \frac{q^{j}}{j !}\right)^{k} \\
& =\frac{1}{k !}(E(q)-1)^{k} \\
& =\frac{1}{k !} \sum_{r=0}^{k}(-1)^{k-r}\left(\begin{array}{l}
k \\
r
\end{array}\right) E(q)^{r} \\
& =\frac{1}{k !} \sum_{r=0}^{k}(-1)^{k-r}\left(\begin{array}{l}
k \\
r
\end{array}\right) \sum_{n=0}^{\infty} p_{r}(n) q^{n}
\end{aligned}
$$

to conclude Equation (4).

Proof of Theorem 1. We combine Equation (2) and Equation (4) to obtain

$$
\begin{aligned}
\sum_{d \mid n} \frac{1}{d} & =\sum_{k=1}^{n} \frac{1}{k} \sum_{r=1}^{k}(-1)^{r}\left(\begin{array}{l}
k \\
r
\end{array}\right) p_{r}(n) \\
& =\sum_{r=1}^{n}(-1)^{r} p_{r}(n) \sum_{k=r}^{n} \frac{1}{k}\left(\begin{array}{l}
k \\
r
\end{array}\right) \\
& =\sum_{r=1}^{n} \frac{(-1)^{r}}{r}\left(\begin{array}{l}
n \\
r
\end{array}\right) p_{r}(n) .
\end{aligned}
$$

Now we can deduce Equation (1) from the fact that $\sum_{d \mid n} \frac{n}{d}=\sum_{j \mid n} j$.

Remark 1. It is interesting to note the following combinatorial identity for $p(n)$ [4],

$$
p(n)=\sum_{r=0}^{n}(-1)^{r}\left(\begin{array}{l}
n+1 \\
r+1
\end{array}\right) p_{r}(n) .
$$

Remark 2. Let $p_{-r}(n)$ be defined by $\sum_{n=0}^{\infty} p_{-r}(n) q^{n}=\prod_{j=1}^{\infty}\left(1-q^{j}\right)^{-r}$. Then it can be proved that

$$
\sum_{d \mid n} d=n \sum_{r=1}^{n} \frac{(-1)^{r-1}}{r}\left(\begin{array}{l}
n \\
r
\end{array}\right) p_{-r}(n)
$$

\section{References}

[1] A. D. Forbes, Congruence properties of functions related to the partition function, Pacific J. Math. 158 (1993), 145-156. 
[2] L. Comtet, Advanced Combinatorics: The Art of Finite and Infinite Expansions, D. Reidel Publishing Co., Dordrecht, 1974.

[3] N. J. Fine, Basic Hypergeometric Series and Applications, American Mathematical Soc., 1988.

[4] S. K. Jha, A formula for the number of partitions of $n$ in terms of the partial Bell polynomials, preprint, 2020. Available at https://arxiv.org/abs/2006.01627.

[5] S. K. Jha, A formula for the $r$-coloured partition function in terms of the sum of divisors function and its inverse, preprint, 2020. Available at https://arxiv.org/abs/2008.03106. 